\title{
Photoconductivity of Colloidal Quantum Dot Films in Plasmonic Nanogaps ${ }^{\dagger}$
}

\author{
Dario Grimaldi, Emil Kelderer *, Andreas Hohenau, Harald Ditlbacher and Joachim R. Krenn \\ Institute of Physics, University of Graz, Universitätsplatz 5, Graz 8010, Austria; \\ andreas.hohenau@uni-graz.at (A.H.); harald.ditlbacher@uni-graz.at (H.D.); \\ joachim.krenn@uni-graz.at (J.R.K.) \\ * Correspondence: emil.kelderer@edu.uni-graz.at \\ + Presented at the 4th International Conference nanoFIS 2020-Functional Integrated nano Systems, \\ Graz, Austria, 2-4 November 2020.
}

Published: 17 December 2020

\begin{abstract}
We investigate the photoconductivity properties of lead sulphide ( $\mathrm{PbS}$ ) quantum dot ensembles in lithographically tailored gold electrodes with smallest gaps of $15 \mathrm{~nm}$. We demonstrate that quantum dots are reliable nanoscale light/current converters and correlate the measured photocurrents to the quantum dot number, the gap voltage and light irradiance. For the latter, we find a photocurrent power law dependence with an exponent of $2 / 3$. Furthermore, we probe the role of plasmonic effects in the gold electrodes and image by scanning photocurrent microscopy the spatial dependence of photocurrent generation.
\end{abstract}

Keywords: quantum dots; photoconductivity; plasmonics

\section{Introduction}

Colloidal quantum dots are attractive building blocks for highly miniaturized and integrated components in optoelectronic applications. Quantum dots are stable single photon emitters with high quantum efficiency and discrete emission wavelengths that can be simply tuned via the quantum dot size. In combination with the rather simple processability at room temperature, this has led to considerable interest in quantum dot photodetectors in recent years [1]. In particular, PbS quantum dot film photodetectors have been realized with record performance in the visible and near infrared spectral range [2]. Here, we investigate highly miniaturized $\mathrm{PbS}$ photodetectors involving only a few to a few hundred quantum dots, aiming at efficient light-to-current conversion with a nanoscale footprint.

\section{Photocurrents by Nanogap Quantum Dots}

We use gold electrodes tailored by electron beam lithography as a controlled platform for characterizing the photocurrents from small $\mathrm{PbS}$ quantum dot ensembles. The electrode gaps filled with quantum dots vary between $15 \mathrm{~nm}$ and $1.5 \mu \mathrm{m}$, the generated currents are in the pA-nA range with $\mathrm{nW}-\mu \mathrm{W}$ light power. The PbS quantum dots are capped with lead halide perovskites (MAPbI 3 ) that enable carrier tunneling between individual quantum dots when deposited as closely packed ensembles by spin coating on the whole substrates supporting the electrode gaps. An exemplary photoconductance I/V curve measured from a $200 \mathrm{~nm}$ gap is depicted in Figure 1a. Figure 1b shows the photocurrent dependence on the exciting light fluence measured from a $1.5 \mu \mathrm{m}$ wide gap, closely following a power law with an exponent of $2 / 3$. 
a

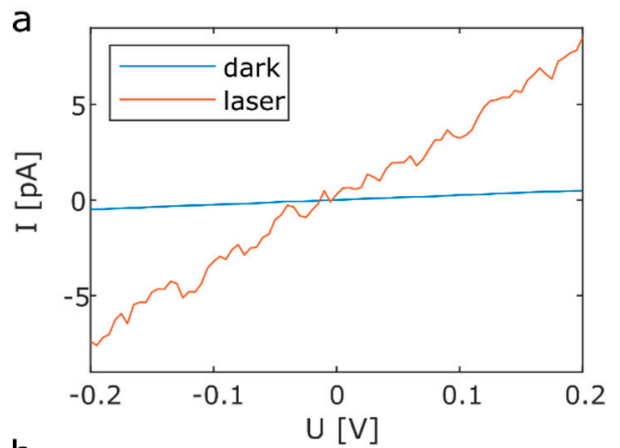

b

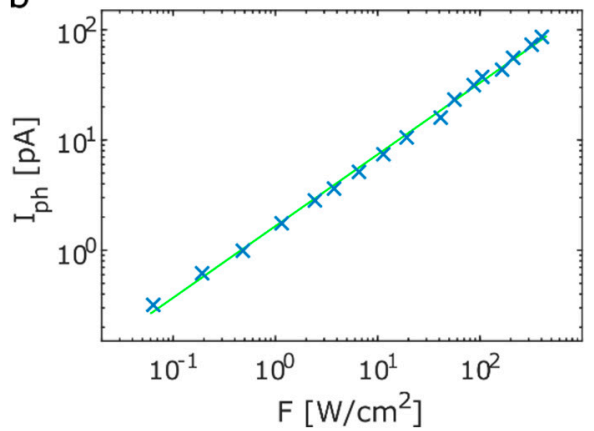

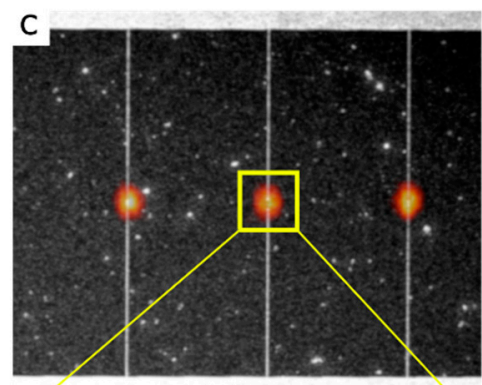

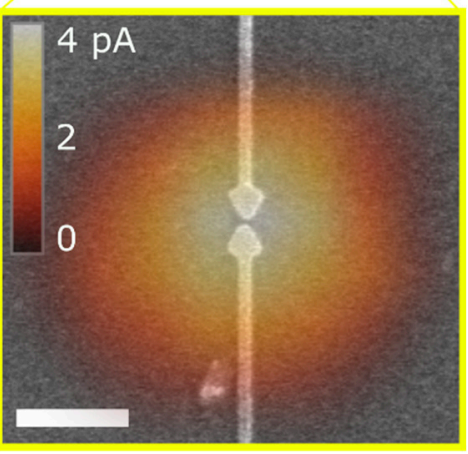

Figure 1. Photocurrent measurements of PbS quantum dots. (a) I/V curves for a $200 \mathrm{~nm}$ gap, the irradiated laser power is $0.5 \mu \mathrm{W}$; (b) measured photocurrent as a function of light fluence (crosses) at a $1.5 \mu \mathrm{m}$ gap with an applied bias of $1 \mathrm{~V}$, the green line is a power law fit with an exponent of 2/3; (c) scanning photocurrent microscope image of a $15 \mathrm{~nm}$ gap, the bias voltage is $0.1 \mathrm{~V}$ and the laser power is $0.2 \mu \mathrm{W}$, the scale bar depicts $500 \mathrm{~nm}$; the laser wavelength is $650 \mathrm{~nm}$ for (a) and (b) and $700 \mathrm{~nm}$ for (c).

\section{Imaging Nanogap Quantum Dots by Scanning Photocurrent Microscopy}

Scanning photocurrent microscopy (SPCM) relies on a focused laser that is scanned over the sample, assigning the measured photocurrent to the scan position. SPCM enables the spatially resolved characterization of photocurrent generation, as illustrated in Figure 1c.

Author Contributions: Investigation, D.G., E.K.; methodology, A.H., H.D.; conceptualization and supervision, J.R.K., A.H. All authors have read and agreed to the published version of the manuscript.

Conflicts of Interest: The authors declare no conflict of interest.

\section{References}

1. Konstantatos, G.; Sargent, E.H. Colloidal quantum dot photodetectors. Infrared Phys. Technol. 2011, 54, 278282.

2. Saran, R.; Curry, R.J. Lead sulphide nanocrystal photodetector technologies. Nat. Photonics 2016, 10, 81-92.

Publisher's Note: MDPI stays neutral with regard to jurisdictional claims in published maps and institutional affiliations.

(C) 2020 by the authors. Licensee MDPI, Basel, Switzerland. This article is an open access article distributed under the terms and conditions of the Creative Commons Attribution (CC BY) license (http://creativecommons.org/licenses/by/4.0/). 